\title{
Comparative effectiveness and safety of direct oral anticoagulants compared to warfarin in morbidly obese patients with acute venous thromboembolism: systematic review and a meta-analysis
}

\author{
Mohamed Nabil Elshafei ${ }^{1} \cdot$ Mouhand F. H. Mohamed $^{2} \cdot$ Ahmed El-Bardissy $^{1} \cdot$ Mohamed Badie Ahmed $^{3}$. \\ Ibtihal Abdallah ${ }^{1} \cdot$ Hazem Elewa $^{4}$ (D) Mohammed Danjuma ${ }^{2,3}$
}

Published online: 18 June 2020

(c) The Author(s) 2020

\begin{abstract}
Direct oral anticoagulant (DOAC) agents are becoming the anticoagulation strategy of choice. However, their use in the treatment of acute venous thromboembolism (VTE) in morbidly obese patients (bodyweight of $>120 \mathrm{~kg}$ or BMI $>40 \mathrm{~kg} /$ $\mathrm{m}^{2}$ ) guarded. This is due to the scarce data supporting their use in this population. As a result, the International Society on Thrombosis and Haemostasis recommended against their use in this cohort of patients. New data emerged supporting the use of DOACs in these patients. Hence, we aimed to systematically review the literature exploring the efficacy and safety of these agents compared to warfarin in VTE treatment in morbidly obese patients. A systematic review of PubMed and EMBASE since inception until 01/04/2020. Subsequently, a non-inferiority (NI of 1.75) meta-analysis utilizing the random-effects model. Five observational studies (6585 patients) were included in our meta-analysis. DOAC analogs were non-inferior compared to warfarin in reducing the primary efficacy outcome of VTE recurrence (OR 1.07, 95\% CI 0.93-1.23) and the primary safety outcome (major bleeding events) (OR 0.80, 95\% CI 0.54-1.17). Our meta-analysis comprising real-world observational data concludes that the use of DOAC analogs in morbidly obese patients (bodyweight of $>120 \mathrm{~kg}$ or BMI $>40 \mathrm{~kg} / \mathrm{m}^{2}$ ) is non-inferior with regards to efficacy and safety compared to warfarin. This finding helps to resolve the uncertainty associated with the use of DOACs in this cohort. Additionally, it invites for a confirmatory non-inferiority randomized controlled trial testing DOAC vs. Warfarin in this group of patients.
\end{abstract}

Keywords Pulmonary embolism · Warfarin · DVT $\cdot$ Morbid obesity · Overweight

Electronic supplementary material The online version of this article (https://doi.org/10.1007/s11239-020-02179-4) contains supplementary material, which is available to authorized users.

Hazem Elewa

Hazem.elewa@qu.edu.qa

Mohamed Nabil Elshafei mohnabil2010@yahoo.com

Mouhand F. H. Mohamed dr.m.oraiby@hotmail.com

Ahmed El-Bardissy aelbardissy@hamad.qa

Mohamed Badie Ahmed ma1510062@qu.edu.qa

Ibtihal Abdallah ibtihal.m.abdallah@gmail.com
Mohammed Danjuma mdanjuma@hamad.qa

Clinical Pharmacy Department, Hamad General Hospital, Doha, Qatar

2 Internal Medicine Department, Hamad Medical Corporation, Doha, Qatar

3 College of Medicine, QU Health, Qatar University, Doha, Qatar

4 College of Pharmacy, QU Health, Qatar University, Doha, Qatar 


\section{Highlights}

- The use of DOACs in the treatment of acute venous thromboembolism (VTE) in morbidly obese patients is controversial.

- There are concerns of decreased drug exposure and under-dosing in the obese patients' population.

- This meta-analysis, aimed to evaluate the effectiveness (rates of VTE events) and safety (major bleeding) of DOAC analogs compared to warfarin in patients with extremely high body weight with acute VTE.

- The project concludes that the use of DOACs in morbidly obese patients (bodyweight of $>120 \mathrm{~kg}$ or BMI $>40 \mathrm{~kg} / \mathrm{m}^{2}$ ) is effective and safe. It supports the current practice of using DOAC analogs as an alternative to warfarin in this cohort of patients.

\section{Introduction}

Venous thromboembolism (VTE) is a prevalent clinical entity affecting approximately 1 to 2 per 1000 patients [1]. These events primarily involve the deep veins of the lower extremities causing deep vein thrombosis (DVT), or embolize to the pulmonary arteries causing a pulmonary embolism (PE). Untreated, PE is associated with a mortality rate that reaches up to $30 \%$ compared with $2-11 \%$ in those treated with anticoagulation (AC) [2-4]. Therefore, prompt identification and treatment initiation with AC therapy is imperative.

Obesity is a worldwide epidemic that drives increasing morbidity and mortality from thrombotic disorders, such as myocardial infarction, stroke, and VTE. It is considered a significant risk factor for VTE by enhancing blood stasis. Studies have shown a significantly increased risk for DVT and $\mathrm{PE}$ in this group of patients [5-7].

In patients with VTE, AC is mandatory to prevent thrombus propagation and recurrence. Low-molecular-weight heparin (LMWH) followed by oral anticoagulation with vitamin $\mathrm{K}$ antagonists (VKA) has been considered the mainstay of therapy until a few years ago [8]. However, as evidenced in variable settings where AC is utilized, warfarin therapy is fraught with a lot of clinical, therapeutic, and logistical issues. These range from potential drug-drug and drugfood interactions, inter and intra-individual variability in both responses to treatment and risk of side effects. Others include the logistics of reliable and robust International normalizing ratio (INR) monitoring [9-12]. Consequent upon these, direct oral anticoagulants (DOACs) have been developed, including factor IIa (thrombin) and factor Xa inhibitors, and introduced to the market. They are approved by the food \& drug administration (FDA) for the management of acute VTE. A steady stream of randomized controlled clinical trials has demonstrated the non-inferiority of these agents when compared to VKA in terms of both efficacy and safety, to reduce of risk of recurrence in patients with both DVT and PE [13-17]. This has resulted in their incorporation into therapeutic national/society guidelines [18]. Since the introduction of DOACs to the market, AC management had encountered significant changes. Current guidelines such as CHEST guidelines suggest DOACs over warfarin in both non-valvular atrial fibrillation (AF) and non-cancer VTE patients $[18,19]$.

Furthermore, DOACs have a wider therapeutic window at fixed dosing regimens, in addition to minimal and manageable food and drug interactions with no requirement for routine monitoring. However, the low representation of obese patients, particularly those with morbid obesity, in the major trials has raised questions about the efficacy, adequacy of fixed dosing, and safety of direct oral anticoagulants in these cohorts of patients. None of the RCTs reported the results of patients with morbid obesity (BMI $\geq 40 \mathrm{~kg} / \mathrm{m}^{2}$ ) [13-17].

There is a scarcity of evidence investigating the efficacy of DOACs in obese patients. In a study of apixaban, bodyweight of more than $120 \mathrm{~kg}$ and body-mass index $[\mathrm{BMI}] \geq 30 \mathrm{~kg} / \mathrm{m}^{2}$ were associated with a lower mean peak concentration and higher volume of distribution compared with average weight [20]. On the contrary, peak concentration, distribution, and half-life of rivaroxaban were similar between patients who weighed more than $120 \mathrm{~kg}$ and those who weighed 70-80 kg [21]. Therefore, because of the concerns of decreased drug exposure and under-dosing in the obese patients' population, the Scientific and Standardization Committee of the International Society on Thrombosis and Hemostasis in its 2016 clinical practice guidelines recommended against the use of DOACs in patients with a BMI of more than $40 \mathrm{~kg} / \mathrm{m}^{2}$ or weighing more than $120 \mathrm{~kg}$ [22]. It also suggested Obtaining drug-specific peak and trough levels to assess therapeutic appropriateness in this population if DOACs are prescribed. Therefore, there is an unresolved uncertainty regarding the utility of DOAC analogs as an acute VTE treatment strategy in morbidly obese patients (BMI $>40 \mathrm{~kg} / \mathrm{m}^{2}$ or weight $>120 \mathrm{~kg}$ ). It will be valuable to demonstrate that DOACs are at least non-inferior to VKA in terms of efficacy and safety in this patient population.

Our meta-analysis, therefore, aimed to evaluate the effectiveness (rates of VTE events) and safety (major bleeding) of DOAC analogs compared to warfarin in patients with extremely high body weight with acute VTE.

\section{Methods}

This review followed PRISMA guidelines [23]. 


\section{Study eligibility criteria}

We attempted to include both real-world observational data and randomized controlled trials that compared DOAC analogs to warfarin in morbidly obese patients (BMI $>40$ or weight $>120 \mathrm{~kg}$ ). At a minimum, studies assessed VTE recurrence or major bleeding events to be included in our review. We excluded studies reporting on pediatrics ( $<18$ years old), as well as studies failing to meet the inclusion criteria.

\section{Search strategy}

We performed an exhaustive literature search of PubMed, Medline, and EMBASE since their inception till 01/04/2020. No language, date, or article type limitations were adopted in our search strategy. Example of a database search strategy is: $(((()((()((($ direct oral anticoagulants) OR (DOAC)) OR (DOACs)) OR (NOAC)) OR (NOACs)) OR (Novel oral anticoagulants)) OR (Rivaroxaban)) OR (Apixaban)) OR (Edoxaban)) OR (Dabigatran)) OR (betrixaban)) AND $(((()((()($ deep venous thromboses[MeSH Terms]) OR (pulmonary embolism[MeSH Terms])) OR (venous thromboembolism[MeSH Terms])) OR (venous thrombosis)) OR (Venous thromboembolism)) OR (VTE)) OR (DVT)) OR (PE)) OR (Deep venous thrombosis)) OR (Pulmonary embolism $)))$ AND ((((((((obese) OR (obesity)) OR (overweight)) OR (Morbid obese)) OR (high BMI)) OR (central obesity[MeSH Terms])) OR (morbid obesity[MeSH Terms])) OR (morbid obesities[MeSH Terms])). Additionally, we attempted a manual reference search of retrieved studies.

\section{Screening and data extraction}

Title and abstract screening were attempted initially. Eligible articles were retrieved for full-text review and assessment for inclusion in our review. Two reviewers (MNE and MFHM) conducted the search and screening. In the case of disagreement not settled by discussion, a third reviewer (AEB) adjudicated the disagreement following the protocol. We used a preplanned template to extract the data. Examples of the data extracted are; general articles information such as the author, publication year, study design, intervention, control, outcome, BMI, weight. Etc.

\section{Outcome}

The primary outcome in our review is the rate of VTE recurrence. Major bleeding events served as our secondary outcome (as defined by the primary study authors). We would look at these outcomes at 6 months of follow-up whenever specified in the study, otherwise the longer duration of observation if no specification provided.

\section{Study quality and risk of bias assessment}

We planned to utilize the Cochrane Collaboration's tool for assessing the risk of bias and quality of randomized controlled trials [24]. Additionally, we used the New castle Ottawa tool to assess the risk of bias assessment of observational studies [25]. We generated funnel plots to screen for publication bias.

\section{Statistical analysis}

The odds ratios (OR) were computed as a measure of effect size. The Forest plot was generated to summarize the results. Additionally, we conducted a sensitivity analysis to screen for consistency and small-study effects. The $\mathrm{I}^{2}$ statistic was used to report heterogeneity. An $\mathrm{I}^{2}>50 \%$ is suggestive of marked heterogeneity in our review. The random-effects model was used as our meta-analytical technique. Understanding that there might be a paucity of studies, we opted for a non-inferiority (NI) margin that corresponds to an OR of 1.75. This NI margin was generated based on a systematic review by Prins et al., and is less than what was used in the EINSTEIN-PE study ( $\mathrm{NI}=2)$, and corresponds to the preservation of at least $75 \%$ of the effect of warfarin over placebo [26, 27]. MetaXl software was used for statistical analysis (version 5.3 (C) EpiGear International Pty Ltd ABN 51134 897411 Sunrise Beach, Queensland, Australia, 2011-2016).

\section{Results}

Our exhaustive search strategy retrieved 475 titles. After screening, five studies were included in our final analysis (Fig. 1 shows the PRISMA flow diagram) [28-32]. The total number of patients evaluated in these studies is 6575 patients. A real-world registry-based retrospective cohort study contributed to the majority of the patients (5780 patients) [32]. All the studies were observational, with the absence of randomized controlled studies meeting our eligibility criteria. Four studies evaluated each of our primary efficacy (VTE recurrence) and safety (major bleeding) outcomes. One study was excluded from the primary efficacy analysis as it reported VTE recurrence in rates [30]. Another study was excluded from the safety outcome as it reported only a composite of major bleeding events and clinically relevant-non-major bleeding events [29]. (Table 1 summary of studies included in the meta-analysis). 

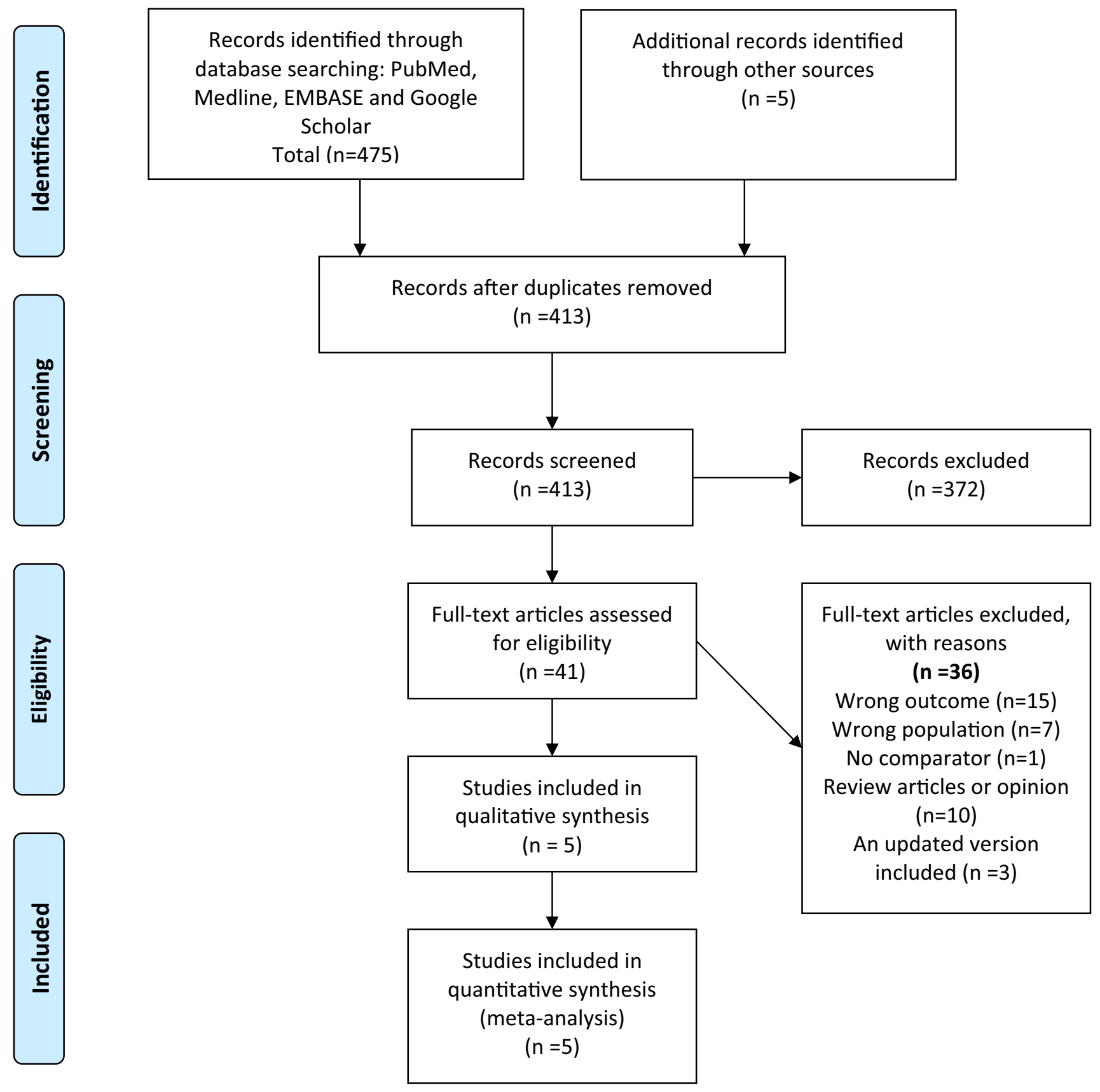

Fig. 1 PRISMA flow diagram

\section{Recurrent venous thromboembolism}

Four studies evaluated VTE recurrent events in morbidly obese patients [28, 29, 31, 32]. These studies showed that DOAC analogs were non-inferior with regards to the primary efficacy outcome of VTE recurrent events (OR 1.07, 95\% CI 0.93-1.23), Q 1.45, I2 0\%. The low $\mathrm{I}^{2}$ suggested the homogeneity of the results (Fig. 2). The funnel plot revealed no marked asymmetry (Fig. 3). Sensitivity analysis showed overall consistency in the final point estimate upon ordered exclusion of the constituent studies; nonetheless, excluding the most extensive study led to a widening of the confidence interval with upper bound crossing the non-inferiority margin (Supplementary material Table 1) [32].

\section{Major bleeding}

Four observational studies evaluated and reported the risk of major bleeding events [28, 30-32]. DOAC analogs had a 


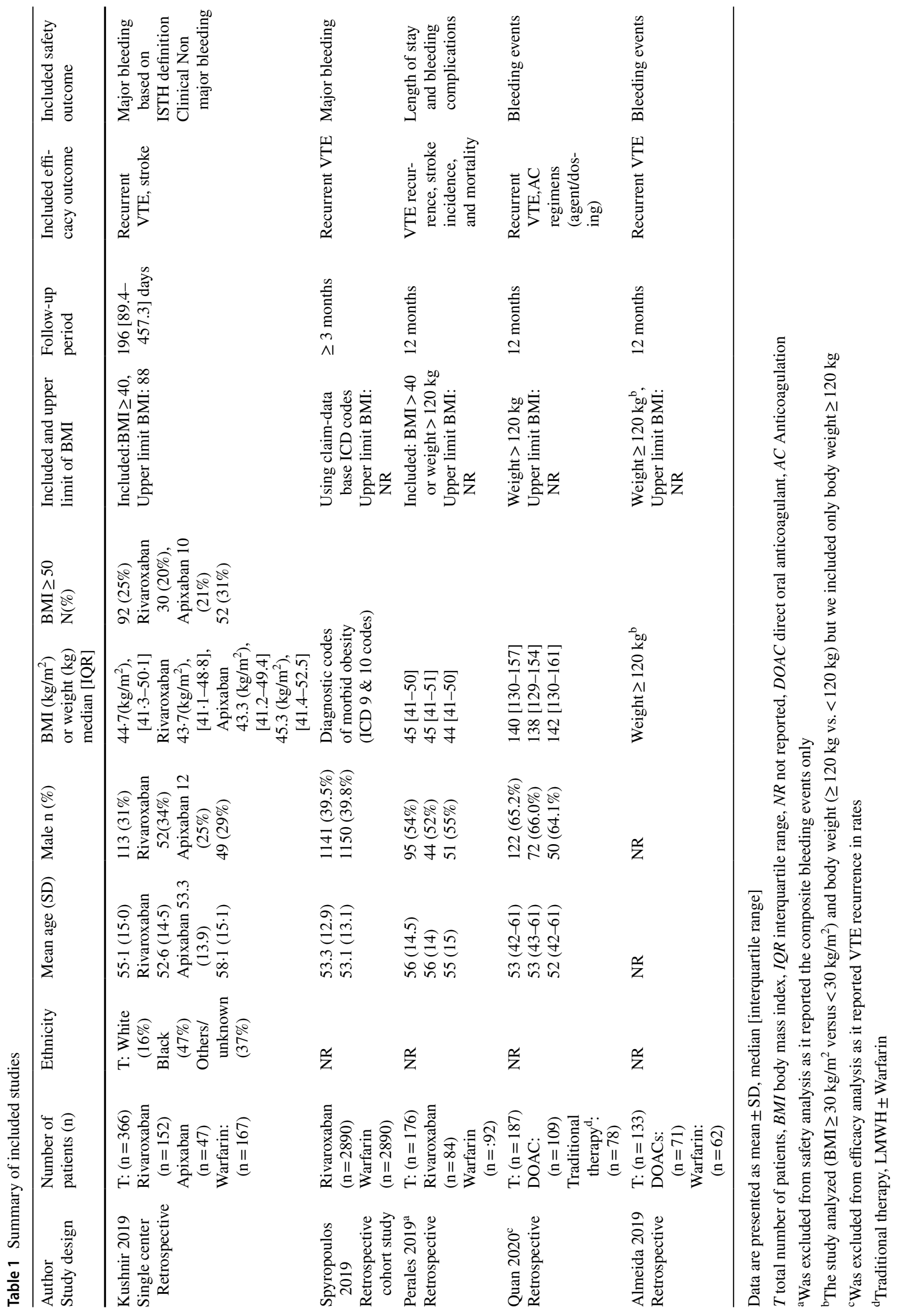



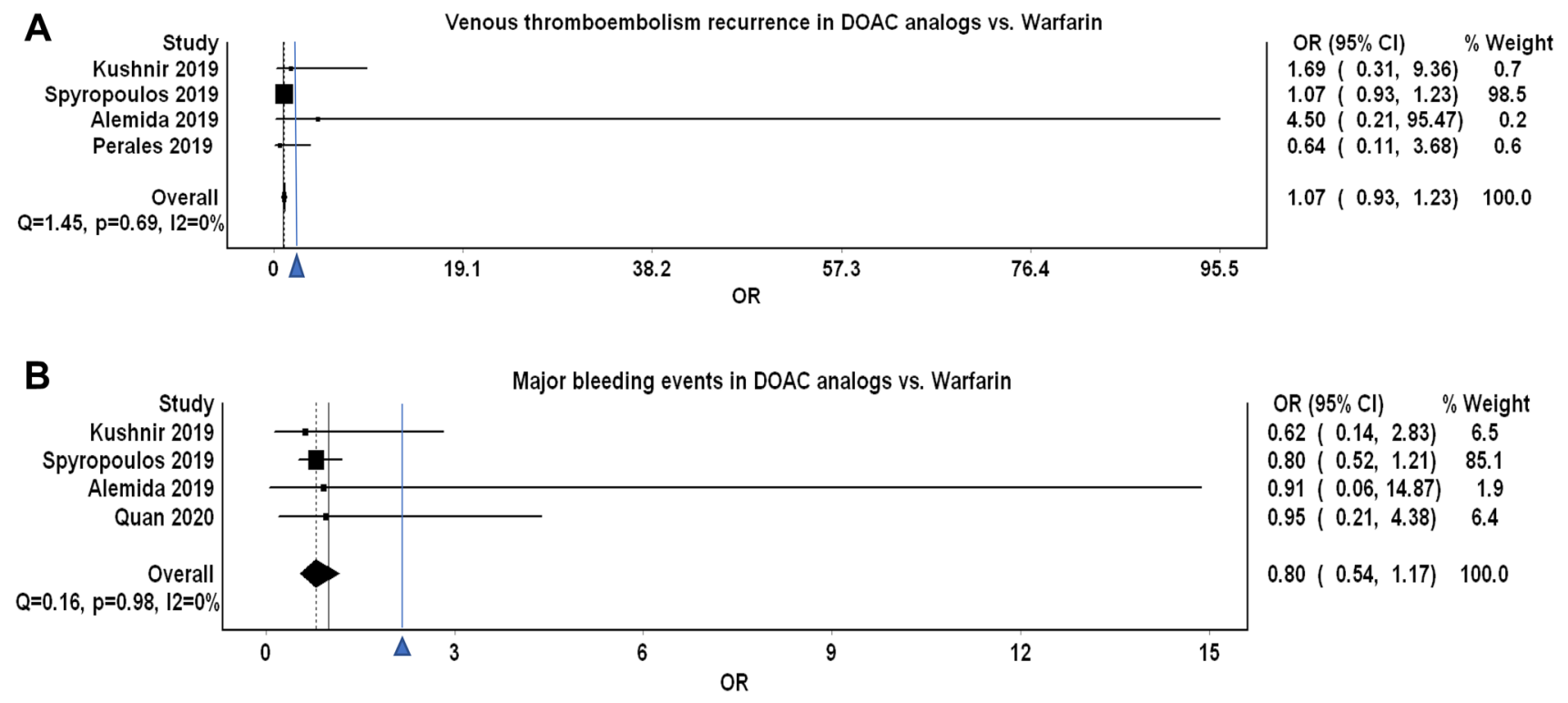

Fig. 2 a Depicting a forest plot of VTE recurrence rates in DOAC analogs compared to warfarin in morbidly obese patients. b Depicting a forest plot of major bleeding events in DOAC analogs compared to warfarin in morbidly obese patients

consistent non-significant trend towards an overall reduced risk of major bleeding events by $20 \%$ (OR $0.80,95 \%$ CI 0.54-1.17, $\mathrm{Q}=0.16, \mathrm{I}^{2}=0 \%$ ) (Fig. 2). The funnel plot showed no marked asymmetry, however, limited by a small number of studies (Fig. 3). Sensitivity analysis did not affect the final point estimate. The exclusion of Spyropoulos et al. resulted in only the widening of the CI (Pooled OR 0.78, 95\% CI 0.28-2.14, Q $=0.16, \mathrm{I}^{2}=0 \%$ ) (Supplementary material Table 1).

\section{Risk of bias assessment}

Most of the included studies were of moderate to high quality $($ NOS $>7$ ) (Supplementary material Table 2 shows a quality assessment of the studies included in the review). The funnel plot showed moderate asymmetry (limited by the small number of trials) (Table 3 ). Hence, publication bias cannot be ruled out.

\section{Discussion}

Obesity is an independent risk factor for the acquisition of VTE. Extremely high BMI has been shown to be correlated with an increased incidence of VTE; this correlation is more apparent with a BMI of $30 \mathrm{~kg} / \mathrm{m}^{2}$ or more [33, 34]. The increased risk of VTE in this cohort is likely due to the increased abdominal pressure and the mechanical effect it exerts on the veins $[35,36]$. Furthermore, the associated molecular hypercoagulable status; this status is postulated to be due to the associated elated levels of tumor necrosis factor-alpha (TNF- $\alpha$ ), transforming growth factor-beta (TGF- $\beta$ ) $[35,36]$. Moreover, the increased levels of Von Willebrand factor, and clotting factors, such as factor VII, factor VIIIc, and fibrinogen [37-39].

The clinical implications (efficacy and safety) of such observations remain uncertain. Subgroup analysis of randomized controlled trials of DOACs in VTE treatment have shown that their efficacy in obese patients $(>100 \mathrm{~kg})$ had no difference compared to average weight [17, 40-42]. However, morbidly obese (bodyweight of $>120 \mathrm{~kg}$ or $\mathrm{BMI}>40 \mathrm{~kg} / \mathrm{m}^{2}$ ) patients were significantly under-represented in these trials. These inconsistencies were the driving factor behind the broad statement of the ISTH recommending against the use of DOACs in extremely high body weight (body weight of $>120 \mathrm{~kg}$ or BMI $>40 \mathrm{~kg} / \mathrm{m}^{2}$ ) [22].

Our meta-analysis, aimed at settling this uncertainty, demonstrated that DOAC analogs are non-inferior to warfarin in terms of effectiveness (VTE events) in morbidly obese patients. Additionally, it showed a propensity towards lower major bleeding events. To the best of our knowledge, our meta-analysis is the first to address the uncertainty regarding the efficacy and safety of DOACs in patients with VTE and extremely high body weight [40-42]. The low $\mathrm{I}_{2}$ and the results of the sensitivity analysis indicated the homogeneity of our data. All the studies included in our review are relatively recent (2019 and 2020). Hence, these were not available to previous reviewers attempting to resolve this uncertainty.

The registry-based study by Spyropoulos et al. 2019 makes up to $88 \%$ of patients included in our review. They 

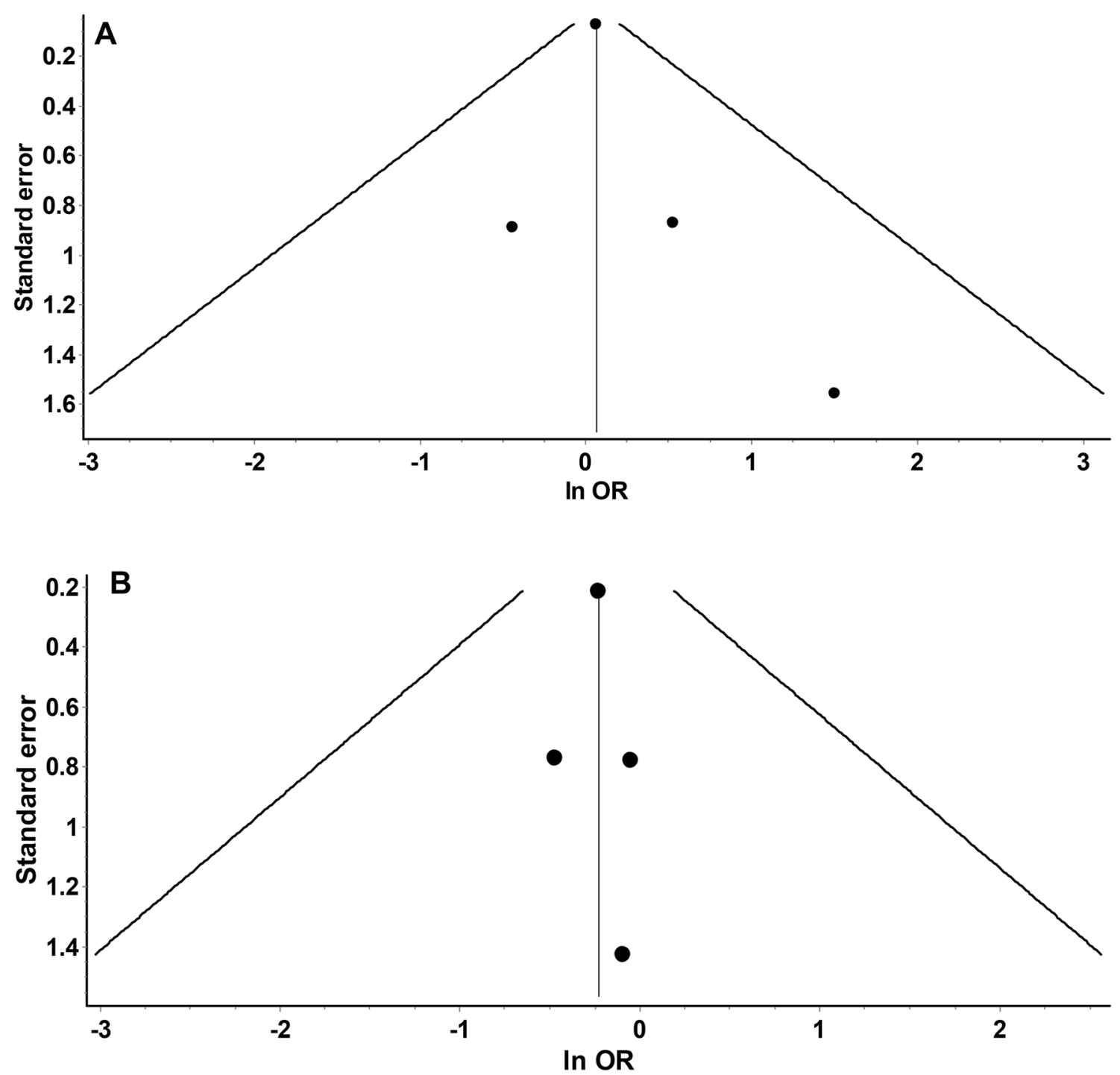

Fig. 3 a Funnel plot to assess the publication bias for studies assessing VTE recurrence in DOAC analogs vs. warfarin displaying no marked asymmetry. b Funnel plot to assess the publication bias for

retrospectively studied rivaroxaban compared to warfarin in an adjusted comparison of 5780 patients. From this realworld analysis, they concluded a similar efficacy (rates of VTE events) and safety (major bleeding events). One major limitation of this study was the use of a claims-coded database. Besides, it did not report the International Normalized Ration (INR), and the time in therapeutic (TTR) for patients on warfarin, thus, bias may have been introduced [32]. Kushiner et al. 2019 investigated the use of rivaroxaban and Apixaban vs. warfarin in morbidly obese patients (BMI of $\geq 40 \mathrm{~kg} / \mathrm{m}^{2}$ ) with atrial fibrillation and DVT. This study included 366 patients and also concluded that the incidence of recurrent VTE and major bleeding did not differ across the three cohorts [28]. This study was limited by missing data for patients' history of thrombotic risk factors and by studies assessing major bleeding events in DOAC analogs vs. warfarin showing no marked asymmetry

the presence of malignancy and bariatric surgery, which might independently contribute to a higher risk of thromboembolism. Additionally, a high proportion of the population was of African American and Hispanic origin, which questions the generalizability of the findings to other racial groups of morbidly obese patients [28].

In early 2020, Coons et al. retrospectively evaluated VTE recurrence and bleeding outcomes in 1840 cases of acute VTE, which were treated with either DOACs or warfarin. Included patients had bodyweight that ranged between 100 and $300 \mathrm{~kg}$. This study did not detect any significant difference in the rate of VTE recurrence between DOACs and warfarin $(6.5 \%$ vs. $6.4 \% ; p=0.93)$. Bleeding occurred in $1.7 \%$ and $1.2 \%$ of patients on DOACs and warfarin, respectively $(\mathrm{p}=0.31)$. However, $50-55 \%$ of the patients in this 
study had a BMI of less than $40 \mathrm{~kg} / \mathrm{m}^{2}$. Although their results support our conclusion, they did not report the outcomes for morbidly obese patients exclusively; hence, their study was excluded from our review [43].

Our meta-analysis is the first meta-analysis that demonstrated the non-inferior effectiveness and safety of DOAC analogs in morbidly obese patients and resolved this uncertainty. It has a good number of patients, out of which the biggest is a registry-based study examining the effect of these agents in real-world settings. Our review is not without limitations. It comprised of observational studies only; it is known that these studies have an inherently higher risk of bias. Secondly, we did not adjust for potential confounders (age, gender, and history of-or active malignancy). Additionally, the major DOAC used in the included studies was rivaroxaban, followed by Apixaban. This limits the generalizability of our findings to other DOAC analogs. Lastly, DOACs dosing information including drug interactions were not reported except for Quan et al. [30].

Acknowledging these limitations, a multicenter randomized controlled trial testing DOAC analogs vs. warfarin in morbidly obese patients is needed to settle this uncertainty once and for all. We think that the use of DOAC analogs as an intervention in this study will be ethically justifiable by the results of our review and the primary studies included in it.

\section{Conclusion}

Our meta-analysis concludes that the use of DOACs in morbidly obese patients (bodyweight of $>120 \mathrm{~kg}$ or $\mathrm{BMI}>40 \mathrm{~kg} / \mathrm{m}^{2}$ ) is effective and safe. It supports the current practice of using DOAC analogs as an alternative to warfarin in this cohort of patients. However, to finally settle this dispute and to support our findings, a randomized controlled trial to confirm the non-inferiority of DOAC analogs vs. warfarin in morbidly obese patients is warranted.

Acknowledgements Open Access funding provided by the Qatar National Library.

Author contributions MNE, MFHM contributed equally to this paper. MNE, MFHM and AE agreed on the review idea. MNE and MFHM performed the initial search, screening and data extraction. AE, MBA, MFHM and MNE extracted and data. MBA and MFHM constructed the tables. MFHM and ME analyzed the data. MFHM constructed the figures. MNE, MFHM, AE, IA wrote the initial manuscript. The manuscript was then critically revised by MBA, MID and HE. All the authors approved the final version of the manuscript for publication.

Funding Apart from publication fund provided by QNL. No other funding sought for the conduct of this review.

\section{Compliance with ethical standards}

Conflict of interest The authors declared that no conflicts of interest with relevant to this review or its publication.

Ethics approval None sought or required as this is a secondary synthesis of an already available data.

Research involving human and animal participants Consent not applicable to this review. However, all authors consented and approved the final manuscript for publication.

Open Access This article is licensed under a Creative Commons Attribution 4.0 International License, which permits use, sharing, adaptation, distribution and reproduction in any medium or format, as long as you give appropriate credit to the original author(s) and the source, provide a link to the Creative Commons licence, and indicate if changes were made. The images or other third party material in this article are included in the article's Creative Commons licence, unless indicated otherwise in a credit line to the material. If material is not included in the article's Creative Commons licence and your intended use is not permitted by statutory regulation or exceeds the permitted use, you will need to obtain permission directly from the copyright holder. To view a copy of this licence, visit http://creativecommons.org/licenses/by/4.0/.

\section{References}

1. Beckman MG, Hooper WC, Critchley SE, Ortel TL (2010) Venous thromboembolism: a public health concern. Am J Prev Med 38(4 Suppl):S495-S501. https://doi.org/10.1016/j.amepr e.2009.12.0172

2. Carson JL et al (1992) The clinical course of pulmonary embolism. N Engl J Med 326(19):1240-1245

3. den Exter PL, van Es J, Klok FA (2013) Risk profile and clinical outcome of symptomatic subsegmental acute pulmonary embolism. Blood 122:1144-1149

4. Goldhaber SZ, Visani L, De Rosa M (1999) Acute pulmonary embolism: clinical outcomes in the International Cooperative Pulmonary Embolism Registry (ICOPER). Lancet 353(9162):1386-1389

5. Ageno $\mathrm{W}$ et al (2008) Cardiovascular risk factors and venous thromboembolism: a meta-analysis. Circulation 117(1):93-102

6. Holst AG, Jensen G, Prescott E (2010) Risk factors for venous thromboembolism: results from the Copenhagen City Heart Study. Circulation 121(17):1896-1903

7. Samama MM (2000) An epidemiologic study of risk factors for deep vein thrombosis in medical outpatients: the Sirius study. Arch Intern Med 160(22):3415-3420

8. Kearon C et al (2012) Antithrombotic therapy for VTE disease: antithrombotic therapy and prevention of thrombosis, 9th ed: American College of Chest Physicians Evidence-Based Clinical Practice Guidelines. Chest 141(2):e419S-e496S

9. Pérez-Andreu V, Roldán V, López-Fernández MF (2010) Pharmacogenetics of acenocoumarol in patients with extreme dose requirements. J Thromb Haemost 8:1012-1017

10. Holbrook AM et al (2005) Systematic overview of warfarin and its drug and food interactions. Arch Intern Med 165(10): 1095-1106

11. Sconce E et al (2007) Vitamin K supplementation can improve stability of anticoagulation for patients with unexplained variability in response to warfarin. Blood 109(6):2419-2423 
12. Ansell J et al (2008) Pharmacology and management of the vitamin K antagonists: American College of Chest Physicians Evidence-Based Clinical Practice Guidelines (8th Edition). Chest 133(6 Suppl):160S-198S

13. Agnelli $\mathrm{G}$ et al (2013) Oral apixaban for the treatment of acute venous thromboembolism. N Engl J Med 369(9):799-808

14. Hokusai VTEI et al (2013) Edoxaban versus warfarin for the treatment of symptomatic venous thromboembolism. N Engl J Med 369(15):1406-1415

15. Investigators $E$ et al (2010) Oral rivaroxaban for symptomatic venous thromboembolism. N Engl J Med 363(26):2499-2510

16. Investigators EP et al (2012) Oral rivaroxaban for the treatment of symptomatic pulmonary embolism. N Engl J Med 366(14):1287-1297

17. Schulman $S$ et al (2009) Dabigatran versus warfarin in the treatment of acute venous thromboembolism. N Engl J Med 361(24):2342-2352

18. Kearon $\mathrm{C}$ et al (2016) Antithrombotic therapy for VTE disease: CHEST guideline and expert panel report. Chest 149(2):315-352

19. Phillippe $\mathrm{HM}$ et al (2016) Pharmacist interventions regarding the appropriateness of apixaban, rivaroxaban, dabigatran, and warfarin in a university-affiliated outpatient clinic. J Pharm Technol $32: 245-252$

20. Upreti VV et al (2013) Effect of extremes of body weight on the pharmacokinetics, pharmacodynamics, safety and tolerability of apixaban in healthy subjects. Br J Clin Pharmacol 76(6):908-916

21. Kubitza D et al (2007) Body weight has limited influence on the safety, tolerability, pharmacokinetics, or pharmacodynamics of rivaroxaban (BAY 59-7939) in healthy subjects. J Clin Pharmacol 47(2):218-226

22. Martin K et al (2016) Use of the direct oral anticoagulants in obese patients: guidance from the SSC of the ISTH. JTH 14(6):1308-1313

23. Moher D et al (2009) Preferred reporting items for systematic reviews and meta-analyses: the PRISMA statement. BMJ 339:b2535

24. Higgins JPT et al (2011) The Cochrane Collaboration's tool for assessing risk of bias in randomised trials. BMJ 343:d5928

25. Lo CK-L, Mertz D, Loeb M (2014) Newcastle-Ottawa Scale: comparing reviewers' to authors' assessments. BMC Med Res Methodol 14(1):45

26. Einstein-PE Investigators (2012) Oral rivaroxaban for the treatment of symptomatic pulmonary embolism. N Engl J Med 366(14):1287-1297

27. Prins MH, Lensing AW (2013) Derivation of the non-inferiority margin for the evaluation of direct oral anticoagulants in the treatment of venous thromboembolism. Thromb J 11(1):13

28. Kushnir M, Choi Y, Eisenberg R et al (2019) Efficacy and safety of direct oral factor Xa inhibitors compared with warfarin in patients with morbid obesity: a single-centre, retrospective analysis of chart data. Lancet Haematol 6(7):e359-e365. https://doi. org/10.1016/S2352-3026(19)30086-9

29. Perales IJ, San Agustin K, DeAngelo J, Campbell AM (2020) Rivaroxaban versus warfarin for stroke prevention and venous thromboembolism treatment in extreme obesity and high body weight. Ann Pharmacother 54(4):344-350. https://doi. org/10.1177/1060028019886092
30. Quan S, Smith J, Wu C, Koshman SL, Nguyen B, Bungard TJ (2020) Anticoagulant therapies and outcomes in obese patients with acute venous thromboembolism. Thromb Res 187:56-62. https://doi.org/10.1016/j.thromres.2020.01.011

31. Sa RA et al (2019) Efficacy and safety of direct oral anticoagulants in obese patients with venous thromboembolism. Blood 134(Supplement_1):3675

32. Spyropoulos AC, Ashton V, Chen YW, Wu B, Peterson ED (2019) Rivaroxaban versus warfarin treatment among morbidly obese patients with venous thromboembolism: comparative effectiveness, safety, and costs. Thromb Res 182:159-166. https://doi. org/10.1016/j.thromres.2019.08.021

33. Lee YR, Blanco DD (2017) Efficacy of standard dose unfractionated heparin for venous thromboembolism prophylaxis in morbidly obese and non-morbidly obese critically ill patients. J Thromb Thrombolysis 44(3):386-391

34. Nejat EJ, Polotsky AJ, Pal L (2010) Predictors of chronic disease at midlife and beyond - the health risks of obesity. Maturitas 65(2):106-111

35. Russolillo A et al (2012) Obesity and psoriatic arthritis: from pathogenesis to clinical outcome and management. Rheumatology 52(1):62-67

36. Sam RC et al (2007) Chronic venous disease in a cohort of healthy UK Asian men. Eur J Vasc Endovasc Surg 34(1):92-96

37. Nain C, Donna S, Gökhan S, Stampfer M, Rimm EB et al (2001) Plasma insulin leptin and soluble levels in relation to obesityrelated atherogenic and thrombogenic cardiovascular disease risk factors among men. Atherosclerosis. https://doi.org/10.1016/ S0021-9150(00)00755-3

38. Steffen LM et al (2009) Metabolic syndrome and risk of venous thromboembolism: Longitudinal Investigation of Thromboembolism Etiology. JTH 7(5):746-751

39. Klarin D, Emdin CA, Natarajan P, Conrad MF, Kathiresan S (2017) Genetic analysis of venous thromboembolism in identifies the ZFPM2 locus and implicates obesity as a causal risk factor. Circ Cardiovasc Genet. https://doi.org/10.1161/CIRCGENETI CS.116.001643

40. Hokusai-VTE Investigators, Büller HR, Décousus H et al (2013) Edoxaban versus warfarin for the treatment of symptomatic venous thromboembolism. N Engl J Med 369(15):1406-1415

41. Einstein-PE Investigators, Büller HR et al (2012) Oral rivaroxaban for the treatment of symptomatic pulmonary embolism. N Engl J Med 366(14):1287-1297

42. EINSTEIN Investigators, Bauersachs R, Berkowitz SD et al (2010) Oral rivaroxaban for symptomatic venous thromboembolism. N Engl J Med 363(26):2499-2510

43. Coons JC, Albert L, Bejjani A, Iasella CJ (2020) Effectiveness and safety of direct oral anticoagulants versus warfarin in obese patients with acute venos thromboembolism. Pharmacotherapy 40(3):204-210. https://doi.org/10.1002/phar.2369

Publisher's Note Springer Nature remains neutral with regard to jurisdictional claims in published maps and institutional affiliations. 